\title{
Small molecule compounds alleviate anisomycin-induced oxidative stress injury in SH-SY5Y cells via downregulation of p66shc and $A \beta 1-42$ expression
}

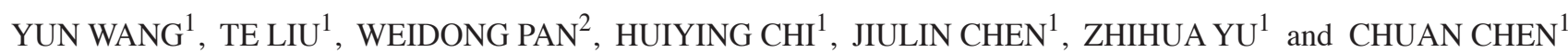 \\ ${ }^{1}$ Shanghai Geriatric Institute of Chinese Medicine, Longhua Hospital, Shanghai University of Traditional \\ Chinese Medicine, Shanghai 200031; ${ }^{2}$ Department of Neurology, Shuguang Hospital Affiliated to \\ Shanghai University of Traditional Chinese Medicine, Shanghai 201203, P.R. China
}

Received January 14, 2015; Accepted September 1, 2015

DOI: $10.3892 /$ etm.2015.2921

\begin{abstract}
Oxidative stress and ageing are important factors contributing to the pathogenesis of Alzheimer's disease (AD), which is associated with neuronal damage and $\beta$-amyloid (A $\beta$ ) deposition. The p66shc adaptor protein is important for the regulation of oxidative stress and ageing. In the present study, SH-SY5Y human neuroblastoma cells were treated with anisomycin in order to establish a cell model of oxidative stress-induced neuronal damage. The results from quantitative polymerase chain reaction, enzyme-linked immunosorbent assay and western blotting demonstrated that anisomycin was able to stimulate the secretion of $\mathrm{A} \beta 1-42$ from SH-SY5Y cells and upregulate the expression levels of p66shc, which was associated with concomitant damage to SH-SY5Y cells. In addition, the protective effects of various small molecule compounds with antioxidant properties against neuronal damage were evaluated. Notably, treatment of SH-SY5Y cells with gallic acid was associated with significant downregulation of p66shc protein expression levels, reduced anisomycin-induced secretion of A $\beta 1-42$, and increased superoxide dismutase activity and acetylcholine secretion levels. The results of the present study suggested that anisomycin is able to promote oxidative neuronal damage by inducing the secretion of $A \beta 1-42$ from neurons and increasing the neuronal expression of p66shc, and this damage may be attenuated by treatment with gallic acid. Therefore, gallic acid and similar small molecule compounds
\end{abstract}

Correspondence to: Professor Chuan Chen or Professor Te Liu, Shanghai Geriatric Institute of Chinese Medicine, Longhua Hospital, Shanghai University of Traditional Chinese Medicine, Building C, 365 Xiangyang Road, Shanghai 200031, P.R. China

E-mail: drchenchuan@163.com

E-mail: teliu79@126.com

Key words: Alzheimer's disease, amyloid- $\beta$, p66shc, anisomycin, SH-SY5Y cells may be considered for the alleviation of neuronal oxidative stress injury in patients with AD.

\section{Introduction}

Alzheimer's disease (AD) is a progressive neurodegenerative disorder that is predominantly characterized by senile plaques (SP), neurofibrillary tangles and regional neuronal loss $(1,2)$. The primary component of SP is $\beta$-amyloid $(\mathrm{A} \beta)$, which has previously been demonstrated to damage synaptic structures and induce neuronal cell death (1-4). In addition, oxidative stress and ageing promote $\mathrm{A} \beta$ production, which has been associated with the occurrence of $\mathrm{AD}(5)$. The p66shc adaptor protein is important for the regulation of cellular senescence and oxidative stress (6-12); under oxidative stress, the brain is more susceptible to damage, as compared with other tissues and organs. In the early stages of AD, oxidative stress occurs prior to the appearance of pathological characteristics, and accelerates neurodegeneration and $A \beta$ formation; thus suggesting that oxidative stress may be involved in the neuropathological process of AD (1-6).

Anisomycin is an antibiotic produced by Streptomyces griseolus, which is capable of binding to the $60 \mathrm{~S}$ ribosomal subunit and blocking polypeptide chain elongation, thereby inhibiting protein synthesis (13-17). As a result, anisomycin exhibits some degree of toxicity towards all cells, including inducing inflammatory responses and oxidative stress injuries that are associated with neuron oxidation and ageing (13-17). As such, anisomycin-induced cell damage may be used to explore the relationship between p66shc-mediated oxidative stress responses and the development and progression of AD. By treating SH-SY5Y cells with anisomycin, Guo et al (13) demonstrated that anisomycin was able to enhance the production of $A \beta$ and increase the expression of presenilin-1 and other ageing-associated proteins.

Astragaloside IV, cinnamic acid, paeoniflorin, and gallic acid are small-molecule compounds with antioxidant properties (2). In the present study, a cell model was established in which $\mathrm{A} \beta$ deposition was induced via exposure of SH-SY5Y cells to anisomycin. Subsequently, the cell model was used 
to verify whether downregulation of p66shc expression via small molecule compounds was able to alleviate anisomycin-induced damage to SH-SY5Y human neuroblastoma cells.

\section{Materials and methods}

Cell culture. The SH-SY5Y human neuroblastoma cells obtained from the Cell Bank of Shanghai Institutes for Biological Sciences (Shanghai, China) were cultured in a routine manner using Dulbecco's modified Eagle's medium (DMEM; Gibco; Thermo Fisher Scientific, Inc., Waltham, MA, USA), supplemented with $10 \%$ fetal bovine serum at $37^{\circ} \mathrm{C}$ in a humidified incubator containing $5 \% \mathrm{CO}_{2}$. The culture medium was changed every 3 days. Upon reaching confluence, the cells were trypsinized (Gibco; Thermo Fisher Scientific, Inc.) and passaged. Cells in the logarithmic growth phase were selected for subsequent experiments.

Model construction. The SH-SY5Y cells were treated with 0,1 , $3,6,12,25,50$ or $100 \mu \mathrm{mol} / 1$ anisomycin (Shanghai Yuanye Bio Co., Ltd., Shanghai, China) for 0, 12, 24 or $48 \mathrm{~h}$. The anisomycin concentration and treatment duration for optimizing SH-SY5Y cell damage and $A \beta$ deposition were selected for construction of the AD model. The cells were trypsinized and seeded into cell culture plates. Four replicate wells were set up for each group. Upon reaching 70 to $80 \%$ confluence, the culture medium was discarded and the cells were incubated with either dimethyl sulfoxide (DMSO; Gibco; Thermo Fisher Scientific, Inc.) or one of the various concentrations of medium-diluted anisomycin. In addition, a control group and blank control group were constructed: The control group was treated with equal volumes of DMSO, and the blank control group was cultured in equal volumes of medium for an additional 24 hours. Cell morphology was observed under a microscope (DMI3000; Leica Microsystems, Inc., Buffalo Grove, IL, USA). At specified times, the cells were centrifuged (Allegra 64R; Beckman Coulter, Inc., Miami, FL, USA) at 1,000 x g for $5 \mathrm{~min}$ at $10^{\circ} \mathrm{C}$, and the resulting supernatant and cell pellets were collected for subsequent assays. After examining relevant indices, an anisomycin concentration of $3 \mu \mathrm{mol} / \mathrm{l}$ was selected. Subsequently, cells were cultured for $0,12,24$ and $48 \mathrm{~h}$ in the presence of anisomycin prior to being harvested and centrifuged. The resulting supernatant and cell pellets were collected for assays.

Screening of small molecule compounds. The SH-SY5Y cells were treated with $500 \mu \mathrm{mol} / 1$ of the small molecule compounds astragaloside IV, cinnamic acid, paeoniflorin and gallic acid (all Shanghai Yuanye Bio Co., Ltd.). Initially, SH-SY5Y cells cultured in petri dishes were subjected to tryptic digestion, and subsequently harvested and seeded into cell culture plates at a density of $1 \times 10^{4}$ cells $/ \mathrm{ml}$, after which the cells were incubated with $3 \mu \mathrm{mol} / 1$ anisomycin for $48 \mathrm{~h}$ in order to establish the AD model. Subsequently, the cells were treated with $50 \mu \mathrm{mol} / 1$ small molecule compounds, which had been diluted in culture medium. In addition, the control group (DMSO-treated group) and blank control group (non-treated group) were constructed. Neither the control group nor the blank control group were treated with small molecule compounds, and the blank control group did not receive anisomycin treatment; instead, an equal volume of culture medium was added to the blank control group, and the cells were cultured for another $24 \mathrm{~h}$. Cell morphology was observed under a phase contrast microscope (DMI3000; Leica Microsystems, Inc.). At predetermined times, the cells were centrifuged, and the resulting supernatant and cell pellets were collected for future assays.

Real time-quantitative polymerase chain reaction ( $R T-q P C R)$. qPCR was conducted according to the method outlined in previous studies $(18,19)$. Briefly, the cell pellets were collected following treatment, after which total RNA was extracted from the cells grown in culture flasks using TRIzol ${ }^{\circledR}$ reagent (Invitrogen; Thermo Fisher Scientific, Inc.), and the concentration $(>10 \mu \mathrm{g} / \mu \mathrm{l})$ and purity $\left(\mathrm{OD}_{260 / 280}>1.80\right)$ of the total RNA were determined. All RNA samples were stored at $-80^{\circ} \mathrm{C}$ prior to analysis. The RNA was reverse transcribed into cDNA using a Reverse Transcription kit (TOYOBO, Co., Ltd., Osaka, Japan), according to the manufacturer's instructions. The resulting cDNA was stored at $-20^{\circ} \mathrm{C}$. RT-qPCR was performed using a MasterCycler RealPlex4 RT-qPCR detection system from Eppendorf (Hamburg, Germany), with the SYBR-Green RealTime PCR Master mix (TOYOBO Co., Ltd.) as the detection dye. RT-qPCR amplification was performed over 40 cycles, with denaturation at $95^{\circ} \mathrm{C}$ for $15 \mathrm{sec}$ and annealing at $58^{\circ} \mathrm{C}$ for $45 \mathrm{sec}$, and a final extension step at $72^{\circ} \mathrm{C}$ for $42 \mathrm{sec}$. The target cDNA was quantified using the relative quantification method. Briefly, a comparative quantification cycle $(\mathrm{Cq})$ was used to determine gene expression levels relative to the $18 \mathrm{~S}$ ribosomal RNA (rRNA), which served as an internal control. The steady state mRNA levels were reported as an $n$-fold difference relative to the internal control. For each sample, the Cq values of the marker genes were normalized using the following equation: $\Delta \mathrm{Cq}=\mathrm{Cq}$ (genes)-Cq(18S rRNA). The relative expression levels were determined using the equation: $\Delta \Delta \mathrm{Cq}=\Delta \mathrm{Cq}$ (sample groups) $-\Delta \mathrm{Ct}$ (control group). The values used to plot the relative expression of markers were calculated using the $2^{-\Delta \Delta C q}$ values. The primers used were as follows: Forward 5'-GTAACCCGTTGAACC CCATT-3', and reverse 5'-CCATCCAATCGGTAGTAGCG-3' for 18S rRNA; and forward 5'-GTATGTGCTCACTGGCTT GC-3', and reverse 5'-CTGACACTTTCAAAGCGGTG-3' for p66.

Western blot analysis. Western blotting was performed according to a method outlined in previous studies $(18,19)$. Briefly, the SH-SY5Y cells were removed from culture flasks using cell scrapers and lysed in precooled $\left(4^{\circ} \mathrm{C}\right)$ cell lysis buffer (Beyotime Institute of Biotechnology, Haimen, China). The protein concentration $(50 \mu \mathrm{g} / \mu \mathrm{l})$ was measured using the bicinchoninic acid assay. Total protein extract $(15 \mu \mathrm{l})$ was separated by $12 \%$ sodium dodecyl sulfate-polyacrylamide gel electrophoresis, and transferred onto polyvinylidene difluoride membranes (EMD Millipore, Billerica, MA, USA). The membranes were then blocked with a solution containing $10 \%$ calf serum, followed by four times washing for 15 min with Tris-buffered saline containing Tween 20 (TBST; Sigma-Aldrich, St. Louis, MO, USA) at room temperature. Subsequently, the membranes were incubated with primary rabbit anti-human p66 (1:1,000; ab87633; Abcam, Shanghai, China) and glyceraldehyde-3-phosphate 


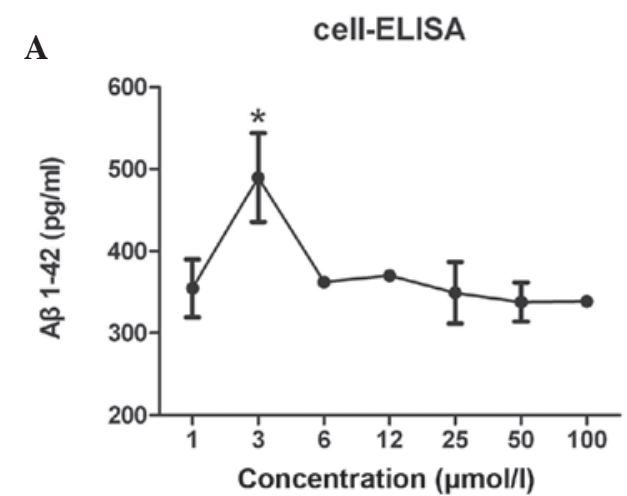

C

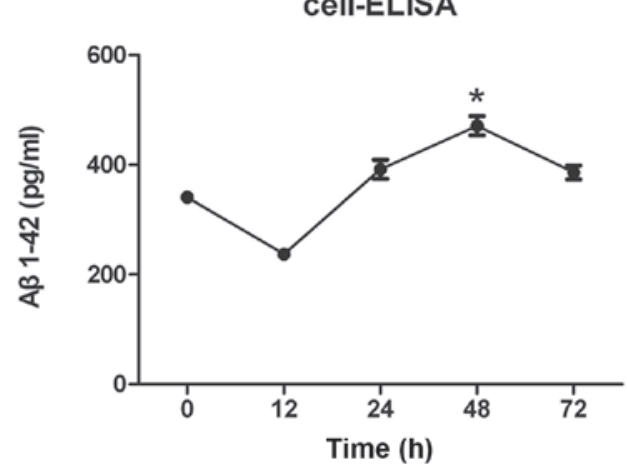

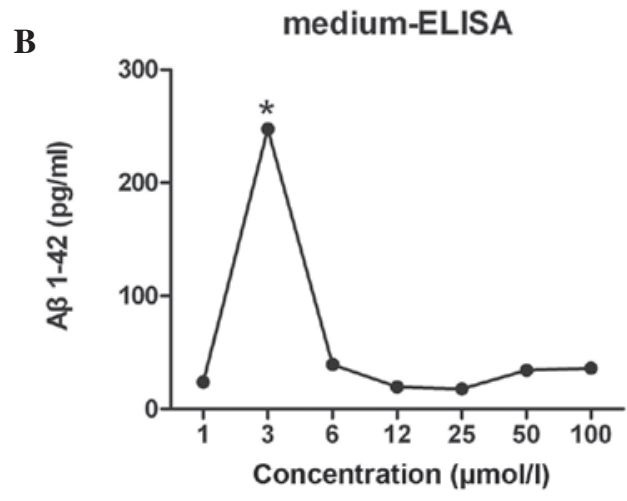

D

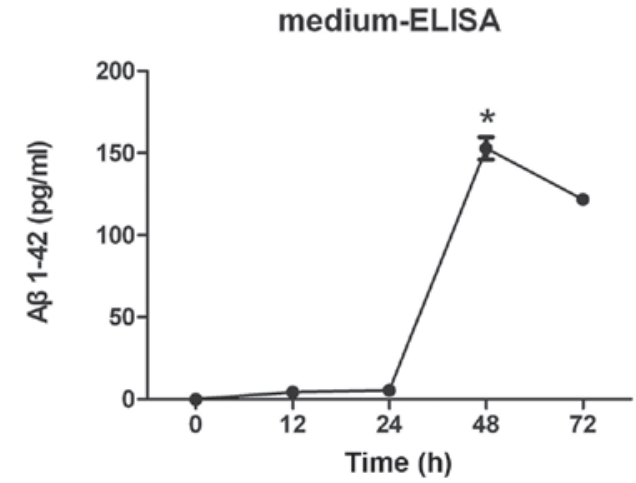

Figure 1. (A) Various concentrations of A $\beta 1-42$ were detected in SH-SY5Y human neuroblastoma cells following treatment of the cells with 1, 3, 6, 12, 25, 50 or $100 \mu \mathrm{mol} / 1$ anisomycin. A $\beta 1-42$ levels in SH-SY5Y cells reached a maximum value following treatment of the cells with $3 \mu \mathrm{mol} / 1$ anisomycin. (B) Various concentrations of secreted A $\beta 1-42$ were detected in the cell culture medium following treatment of SH-SY5Y cells with 1,3,6,12, 25, 50 or 100 $\mu \mathrm{mol} / \mathrm{l}$ anisomycin. The level of secreted A $\beta 1-42$ in cell culture supernatant reached a peak following treatment of SH-SY5Y cells with $3 \mu$ mol/1 anisomycin. (C) Treatment of SH-SY5Y cells with $3 \mu \mathrm{mol} / 1$ anisomycin for $0,12,24,48$ or $72 \mathrm{~h}$ resulted in various A $\beta 1-42$ concentrations in the cells. The A $\beta 1-42$ concentration in SH-SY5Y cells reached a maximum value following stimulation with anisomycin for $48 \mathrm{~h}$. (D) Stimulation of SH-SY5Y cells with $3 \mu \mathrm{mol} / \mathrm{l}$ anisomycin for $0,12,24,48$ or $72 \mathrm{~h}$ resulted in various levels of secreted $\mathrm{A} \beta 1-42$ into the cell culture supernatant. The level of secreted A $\beta 1-42$ in cell culture supernatant reached a peak following $48 \mathrm{~h}$ of treatment with $3 \mu \mathrm{mol} / 1$ anisomycin. Data are presented as the mean \pm standard deviation of triplicate experiments. ${ }^{*} \mathrm{P}<0.05$ vs. 0 h. $\mathrm{A} \beta, \beta$-amyloid.

A

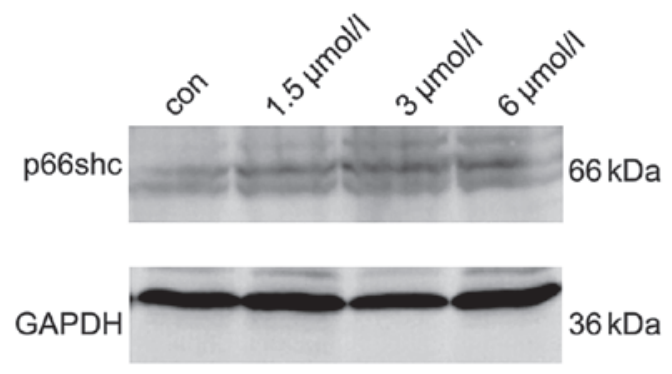

B

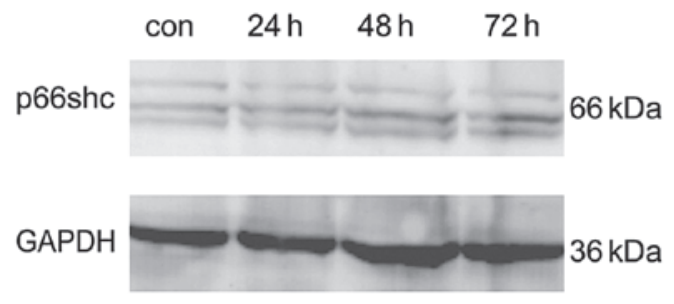

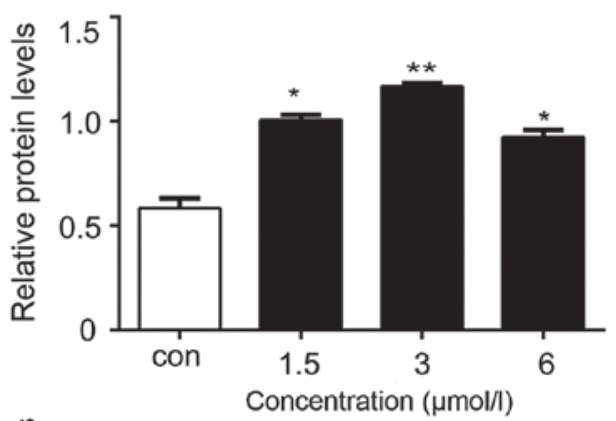

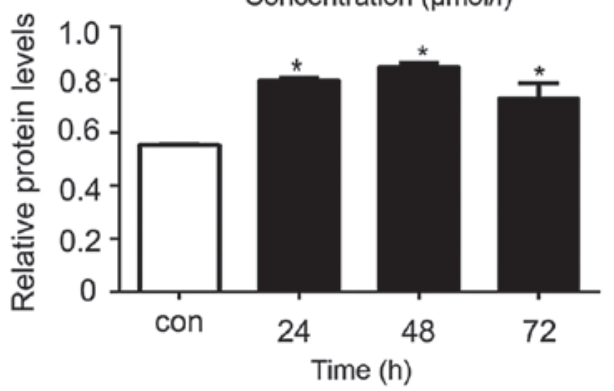

Figure 2. (A) Treatment of the SH-SY5Y human neuroblastoma cells with various concentrations of anisomycin (1.5, 3, or $6 \mu$ mol/1) increased the p66shc protein expression levels. The p66shc protein expression levels peaked when the SH-SY5Y cells were treated with $3 \mu$ mol/1 anisomycin. (B) Stimulation of SH-SY5Y cells with $3 \mu \mathrm{mol} / 1$ anisomycin for 24,4 or $72 \mathrm{~h}$ increased the p66shc protein expression levels, which peaked after $48 \mathrm{~h}$ of stimulation. Data are presented as the mean \pm standard deviation. ${ }^{*} \mathrm{P}<0.05$ vs. the control group; ${ }^{* *} \mathrm{P}<0.01$ vs. the control group. 
A

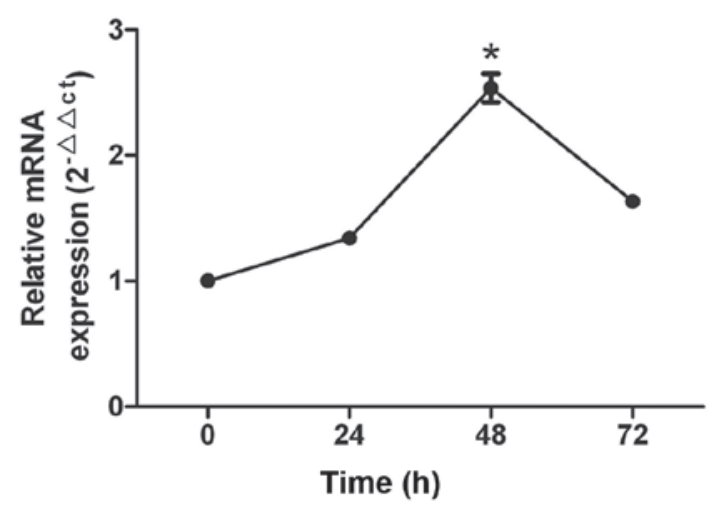

C

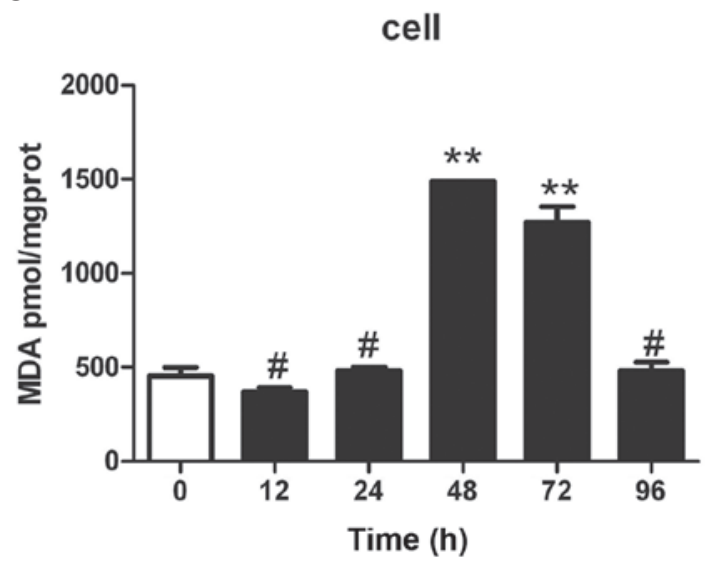

B

cell

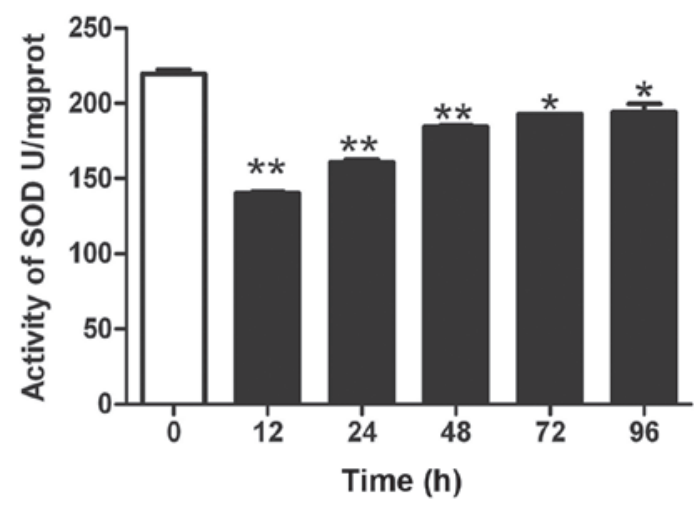

D

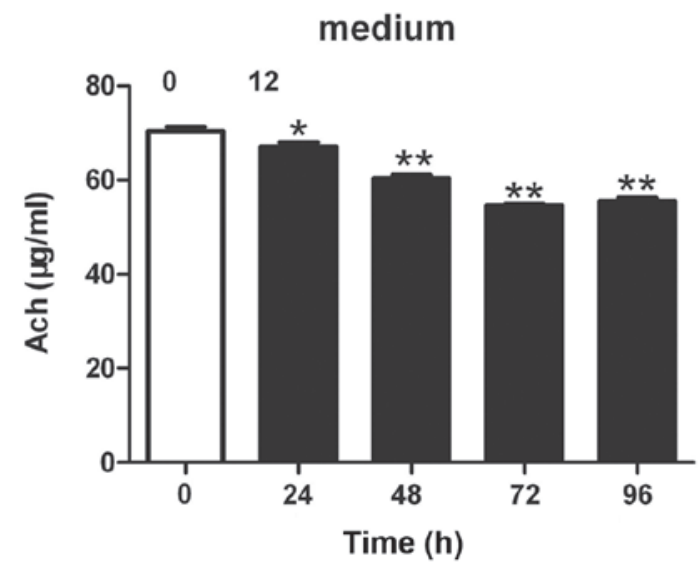

Figure 3. (A) Treatment of SH-SY5Y human neuroblastoma cells with $3 \mu \mathrm{mol} / \mathrm{l}$ anisomycin was associated with significant changes in p66shc mRNA expression levels. The p66shc mRNA expression levels increased by 2.5 -fold after $48 \mathrm{~h}$ of anisomycin treatment, as compared with $0 \mathrm{~h}$. (B) Treatment of SH-SY5Y cells with $3 \mu \mathrm{mol} / 1$ anisomycin for various lengths of time $(0,12,24,48,72$ and $96 \mathrm{~h})$ resulted in significantly reduced SOD activity in the cells, as compared with $0 \mathrm{~h}$ of treatment. The decrease in SOD activity was more evident after 12, 24 and $48 \mathrm{~h}$ of anisomycin treatment. (C) SH-SY5Y cells were treated with $3 \mu \mathrm{mol} / 1$ anisomycin for various lengths of time $(0,12,24,48,72$ and $96 \mathrm{~h})$. The protein expression levels of MDA were significantly increased after 48 and $72 \mathrm{~h}$ of treatment, as compared with $0 \mathrm{~h}$. (D) SH-SY5Y cells were treated with $3 \mu \mathrm{mol} / 1$ anisomycin for various lengths of time ( 0 , $24,48,72$ and $96 \mathrm{~h})$ and the levels of Ach secreted from the cells were significantly reduced after 24, 48, 72 and $96 \mathrm{~h}$, as compared with $0 \mathrm{~h}$. Data are presented as the mean \pm standard deviation of triplicate experiments. ${ }^{\#} \mathrm{P}>0.05$ vs. $0 \mathrm{~h} ;{ }^{*} \mathrm{P}<0.05$ vs. $0 \mathrm{~h} ;{ }^{* *} \mathrm{P}<0.01$ vs. $0 \mathrm{~h}$. SOD, superoxide dismutase; MDA, malondialdehyde; Ach, acetylcholine.

dehydrogenase $(1: 1,000 ; 5174$, Cell Signaling Technology, Inc., Danvers, MA, USA) polyclonal antibodies at $4^{\circ} \mathrm{C}$ overnight. Following extensive washing, the membranes were incubated with secondary peroxidase-conjugated goat anti-rabbit immunoglobulin G antibodies (1:1,000; sc-2004; Santa Cruz Biotechnology, Inc., Dallas, TX, USA) for $1 \mathrm{~h}$. Following further washing steps (15 min each) with TBST at room temperature, the target proteins were visualized by enhanced chemiluminescence (ECL kit; Pierce Biotechnology, Inc., Rockford, IL, USA) and exposure to Kodak Biomax XAR-5 films (Sigma-Aldrich).

Quantification of A $\beta 1-42$ levels by ELISA. The cell pellets were suspended in cell lysis buffer and a double-antibody sandwich ELISA was performed, according to the manufacturer's instructions (Human A $\beta 1-42$ ELISA kit; cat no. h022931; Westang Bio, Shanghai, China). The primary antibody working solution, enzyme-labeled antibody working solution, substrate working solution and stop solution, were added sequentially to the cell lysates. The A $\beta 1-42$ concentration was calculated according to the absorbance values of a standard curve.

Quantification of levels of superoxide dismutase (SOD), malondialdehyde (MDA), and acetylcholine (Ach). The SH-SY5Y cells and their supernatants were collected, and cell pellets were suspended in buffer solution (phosphate-buffered saline or physiological saline; $0.3-0.5 \mathrm{ml}$ per $10^{6}$ cells). Cells were lysed in an ice bath using sonication, in which the tip of an ultrasonic probe was immersed in the liquid. Enzyme and substrate working solutions were then added sequentially. The absorbance of various concentrations of the standard compounds was determined. The SOD, MDA and Ach concentrations were calculated by comparing the absorbance of the samples to those of the standards. The SOD Assay kit, MDA Assay kit, and Ach Assay kit were all purchased from Westang Bio (Shanghai, China).

Statistical analysis. Data are presented as the mean \pm standard deviation. Differences were evaluated using the Student's t-test 
p66shc-cells

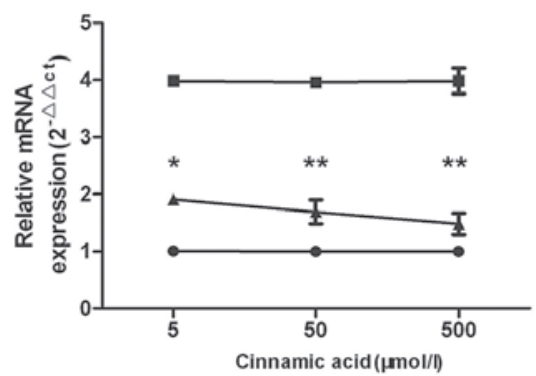

p66shc-cells

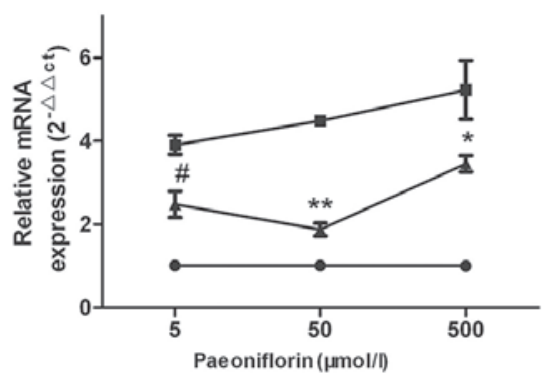

p66shc-cells

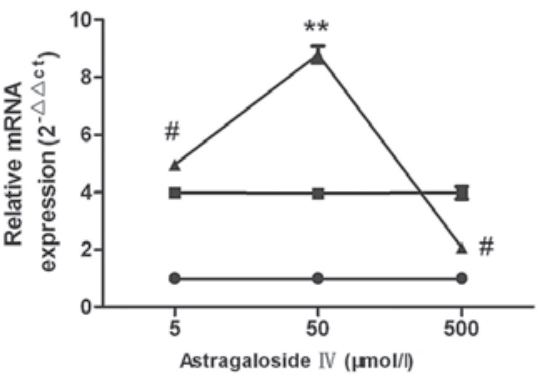

p66shc-cells

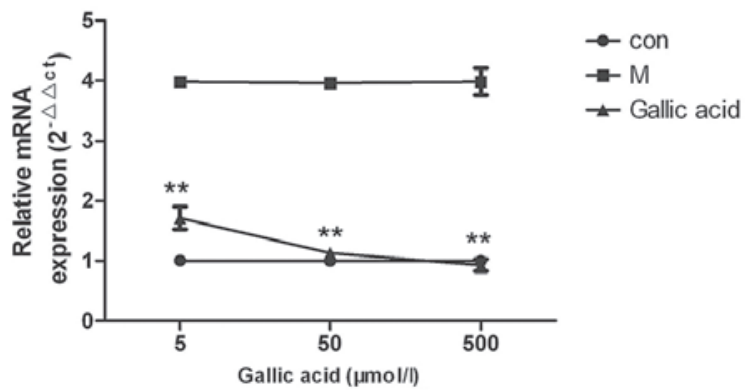

Figure 4. Various concentrations $(5,50$ or $500 \mu \mathrm{mol} / \mathrm{l})$ of cinnamic acid, paeoniflorin and gallic acid significantly reduced the expression levels of p66shc mRNA. At a concentration of $500 \mu \mathrm{mol} / 1$, astragaloside IV decreased the p66shc mRNA expression levels in the cell model. Cinnamic acid and gallic acid had the most significant effects on the mRNA expression levels of p66shc. Data are presented as the mean \pm standard deviation. " $\mathrm{P}>0.05$ vs. the model group; ${ }^{*} \mathrm{P}<0.05$ vs. the model group; ${ }^{* *} \mathrm{P}<0.01$ vs. the model group Con, the control group; $\mathrm{M}$, the model group.
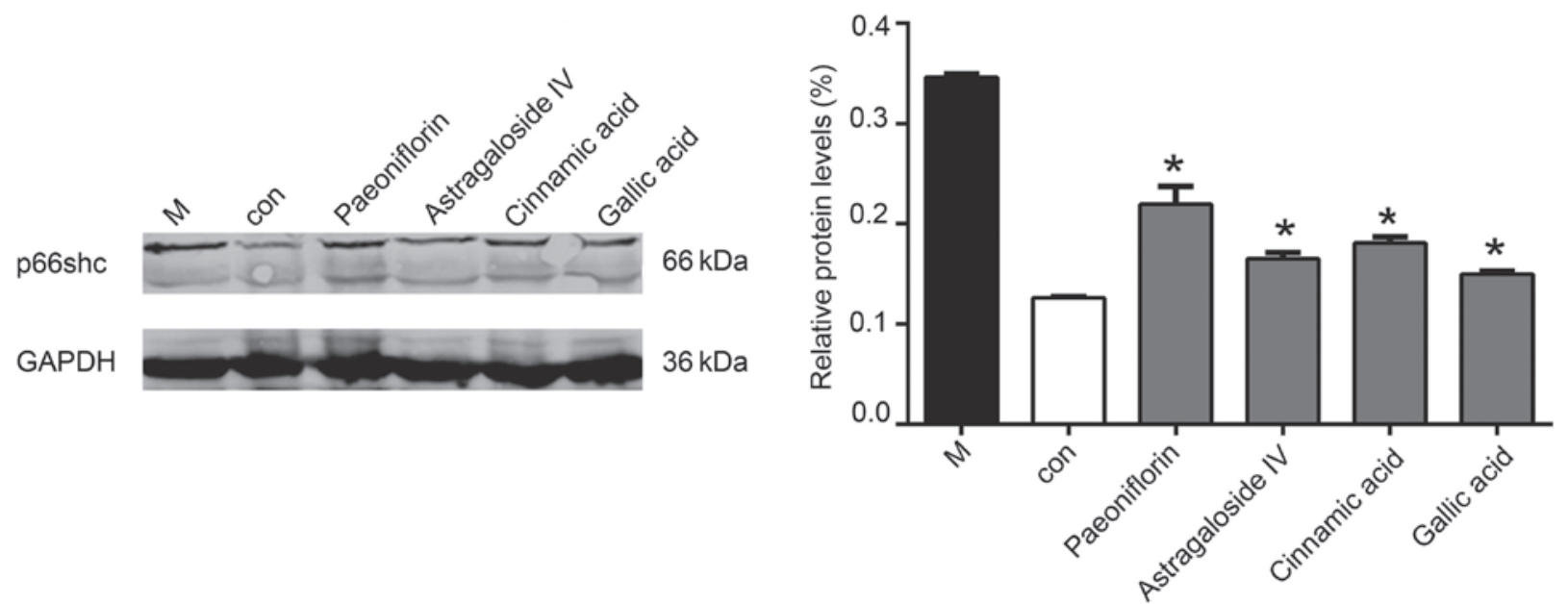

Figure 5. The cell model was treated with $50 \mu \mathrm{mol} / 1$ cinnamic acid, astragaloside IV, paeoniflorin or gallic acid. Gallic acid treatment most significantly reduced the p66shc protein expression levels. Data are presented as the mean \pm standard deviation. "P $<0.05$ vs. the model group. Con, the control group; $\mathrm{M}$, the model group.

and GraphPad Prism 5.0 (GraphPad Software, Inc., La Jolla, CA, USA) was used for statistical analysis. $\mathrm{P}<0.05$ was considered to indicate a statistically significant difference.

\section{Results}

Anisomycin increases the expression levels of A $\beta 1-42$ in SH-SY5Y cells. The levels of A $\beta 1-42$ in the SH-SY5Y cell pellets and culture supernatants were determined using an
ELISA. The A $\beta 1-42$ levels in the SH-SY5Y cells reached a maximum value following treatment of the cells with $3 \mu \mathrm{mol} / 1$ anisomycin (Fig. 1A and B), and this occurred after $48 \mathrm{~h}$ of stimulation (Fig. 1C and D).

Anisomycin increases the expression levels of p66shc in SH-SY5Y cells. The western blot analysis demonstrated that the protein expression levels of p66shc in the SH-SY5Y cells significantly increased following treatment with various 
A

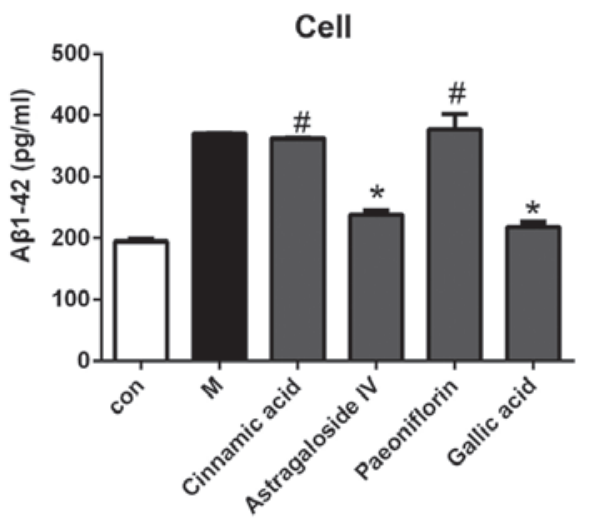

B

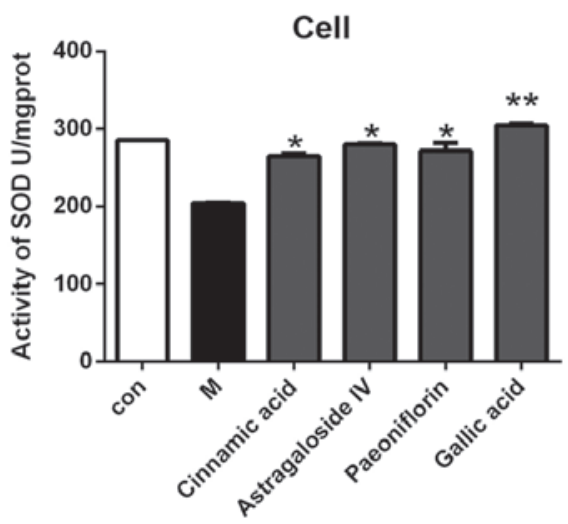

C

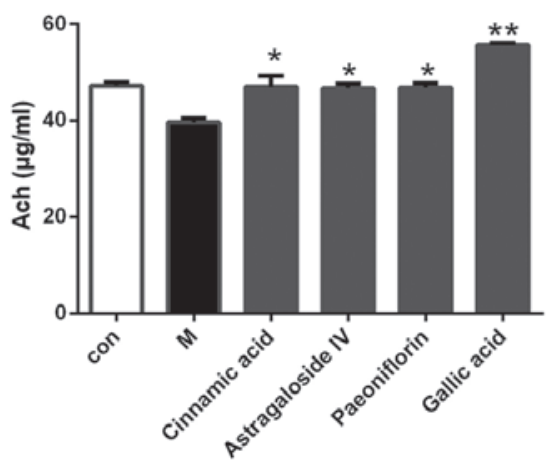

Figure 6. (A) As compared with the cells in the model group, A $\beta$ secretion was reduced following treatment with $50 \mu \mathrm{mol} / 1$ astragaloside IV or gallic acid, with the effects of gallic acid being more significant. (B) The cell model was treated with $50 \mu \mathrm{mol} / 1$ cinnamic acid, astragaloside IV, paeoniflorin or gallic acid. As compared with the untreated model group, the SOD activity was significantly elevated following treatment with the small molecule compounds. (C) As compared with the untreated model group, the Ach level was elevated after treatment with $50 \mu \mathrm{mol} / 1$ cinnamic acid, astragaloside IV, paeoniflorin, or gallic acid. The effects of gallic acid on $\mathrm{A} \beta$ secretion, SOD activity, and Ach secretion, were the most significant of all the small molecule compounds. Data are presented as the mean \pm standard deviation. ${ }^{\prime \prime} \mathrm{P}>0.05$ vs. the model group; ${ }^{*} \mathrm{P}<0.05$ vs. the model group; ${ }^{* *} \mathrm{P}<0.01$ vs. the model group. $\mathrm{A} \beta, \beta$-amyloid; SOD, superoxide dismutase; Ach, acetylcholine, Con, the control group; $\mathrm{M}$, the model group.

concentrations of anisomycin $(1.5,3$ or $6 \mu \mathrm{mol} / \mathrm{l})$, as compared with the control group $(\mathrm{P}<0.05$; Fig. $2 \mathrm{~A})$. The increase in p66shc protein expression levels was most significant when the cells were treated with $3 \mu \mathrm{mol} / 1$ anisomycin $(\mathrm{P}<0.01$; Fig. $2 \mathrm{~A})$. Compared with the p66shc levels at $0 \mathrm{~h}$, the protein expression levels of p66she were significantly increased at various time points $(24,48$ and $72 \mathrm{~h}$ ) following treatment of SH-SY5Y cells with $3 \mu \mathrm{mol} / 1$ anisomycin ( $\mathrm{P}<0.05$; Fig. 2B). The p66shc protein expression levels peaked after $48 \mathrm{~h}$ of treatment with $3 \mu \mathrm{mol} / 1$ anisomycin (P<0.01; Fig. 2B).

Anisomycin increases the mRNA expression levels of p66shc in SH-SY5Y cells. The results of the qPCR demonstrated that the mRNA expression levels of p66shc were significantly increased in the SH-SY5Y cells following treatment with $3 \mu \mathrm{mol} / 1$ anisomycin, in a time-dependent manner $(\mathrm{P}<0.01$; Fig. 3A), as compared with the mRNA expression levels at $0 \mathrm{~h}$. The differences in the threshold cycle (Ct) values between p66shc mRNA and 18S rRNA were compared. The mRNA expression levels of p66shc increased 2.5 fold following treatment of SH-SY5Y cells with $3 \mu \mathrm{mol} / 1$ anisomycin for $48 \mathrm{~h}$, as compared with the mRNA expression levels at $0 \mathrm{~h}$. $(\mathrm{P}<0.01$; Fig. 3A). These results suggest that anisomycin-induced damage in SH-SY5Y cells may be associated with significantly increased p66shc expression.

Anisomycin decreases the activity of SOD and increases the levels of MDA in SH-SY5Y cells. SOD activity was significantly reduced in a time-dependent manner in the SH-SY5Y cells following treatment with $3 \mu \mathrm{mol} / 1$ anisomycin, as compared with SOD activity at $0 \mathrm{~h}(\mathrm{P}<0.01$; Fig. 3B), and this was most pronounced after 12,24 , and $48 \mathrm{~h}$ of anisomycin treatment $(\mathrm{P}<0.01)$. In addition, the protein expression levels of MDA in the SH-SY5Y cells were significantly increased after 48 and $72 \mathrm{~h}$ of anisomycin treatment, as compared with $0 \mathrm{~h}$ of treatment ( $\mathrm{P}<0.01$; Fig. 3C).

Anisomycin reduces the levels of Ach in cell culture supernatants. The levels of Ach secreted by the SH-SY5Y cells significantly decreased over time following treatment of the cells with $3 \mu \mathrm{mol} / 1$ anisomycin, as compared with the levels at $0 \mathrm{~h}(\mathrm{P}<0.01$; Fig 3D). The most significant decreases in Ach secretion occurred following 48, 72 and $96 \mathrm{~h}$ treatment with anisomycin $(\mathrm{P}<0.01)$.

Screening of the small molecule compounds cinnamic acid, astragaloside IV, paeoniflorin and gallic acid. The results of the qPCR demonstrated that various concentrations $(5,50$ or $500 \mu \mathrm{mol} / \mathrm{l}$ ) of cinnamic acid, paeoniflorin and gallic acid were able to reduce the p66shc mRNA expression levels, as compared with the model group ( $\mathrm{P}<0.05$; Fig. 4$)$, whereas, only $500 \mu \mathrm{mol} / 1$ astragaloside IV was able to decrease the p66shc mRNA expression levels in the cell model $(\mathrm{P}<0.05)$. Of the four compounds screened, cinnamic acid and gallic acid exerted the most pronounced effects and decreased the p66shc mRNA expression levels to those that resembled the mRNA expression levels of the cells in the normal group (Fig. 4).

Western blotting demonstrated that $50 \mu \mathrm{mol} / 1$ cinnamic acid, paeoniflorin or gallic acid reduced the protein expression levels of p66shc, although the effects of gallic acid were the most significant $(\mathrm{P}<0.05$; Fig. 5).

As compared with the model group, treatment with $50 \mu \mathrm{mol} / 1$ cinnamic acid and paeoniflorin exerted no significant effects on the secretion of A $\beta 1-42$ from the SH-SY5Y cells, whereas treatment with $50 \mu \mathrm{mol} / 1$ astragaloside IV or gallic acid was associated with decreased $A \beta 1-42$ secretion levels; however, the effects of gallic acid were more 
significant $(\mathrm{P}<0.05$; Fig. $6 \mathrm{~A})$. As compared with the model group, treatment of the cells with $50 \mu \mathrm{mol} / 1$ cinnamic acid, astragaloside IV, paeoniflorin or gallic acid increased the SOD activity and Ach expression levels in the model cells, with the effects of gallic acid being particularly significant $(\mathrm{P}<0.01$; Fig. 6B and C).

\section{Discussion}

The pathogenesis of AD is very complex. Currently, the $\mathrm{A} \beta$ hypothesis, in which $\mathrm{A} \beta$ destroys synaptic structures and impairs hippocampal long-term potentiation, resulting in a decline in learning and memory functions, is widely accepted (17). Extracellular $A \beta$ is associated with neuronal dysfunction by permeabilising lipid bilayers, reducing membrane fluidity and inducing the inflammatory cascade and oxidative stress (1-6).

Previous studies have associated the loss of p66shc with reduced levels of reactive oxygen species and oxidative stress responses in the brain, improved spatial learning and memory, and prevention of ageing-induced behavioral changes (7-13,20,21). In addition, p66shc has been associated with an increased lifespan, which is predominantly reflected in the reduction of brain atrophy, the maintenance of behavioral plasticity and the increase in the overall level of brain-derived neurotrophic factor (22). Furthermore, Sone et al (23) demonstrated a positive correlation between the gene expression levels of p66shc and ageing, and this correlation was particularly evident in the brain.

The levels of p66shc have been associated with oxidation and ageing of the nervous system (10). AD is a neurodegenerative disorder, in which $A \beta$ formation represents an important mechanism underlying its pathogenesis; therefore, compounds that are able to reduce the production of $A \beta$ in neurons and block cell damage may be considered in the prevention and treatment of patients with AD.

The results of the present study demonstrated that anisomycin-induced neuronal damage increased A $\beta 1-42$ secretion, and this was associated with concomitant upregulation of p66shc expression, an increase in the cellular response to oxidative stress, and a reduction in neurotransmitter production. Thus suggesting that anisomycin may induce neuronal damage and increase cellular deposition of A $\beta 1-42$ via an oxidative stress pathway.

Among the four small molecule compounds examined in the present study, gallic acid was able to downregulate p66shc expression in the SH-SY5Y cells, and reduce anisomycin-induced A $\beta$ deposition, enhance SOD activity and increase Ach secretion. These results suggested that gallic acid was able to reduce anisomycin-induced $A \beta$ deposition in the SH-SY5Y cells via the downregulation of p66shc expression. In addition, cinnamic acid, astragaloside IV and paeoniflorin were able to suppress the expression of p66shc, and were able to attenuate anisomycin-induced A $\beta$ deposition. Astragaloside IV reduced $A \beta$ secretion from cells, possibly via mechanisms other than downregulation of p66shc. Although cinnamic acid and paeoniflorin were unable to directly decrease $A \beta$ secretion, they did reduce the oxidative stress responses to varying extents and enhanced neurotransmitter production; thus suggesting that they were able to exhibit minor neuroprotective effects, although these were less significant than those detected for gallic acid.

In conclusion, the present study demonstrated that treatment of SH-SY5Y human neuroblastoma cells with anisomycin was associated with nerve damage and increased $\mathrm{A} \beta$ secretion. In addition, gallic acid was able to downregulate p66shc expression in SH-SY5Y cells, which may have reduced anisomycin-induced $\mathrm{A} \beta$ deposition; thus suggesting that gallic acid exerted protective effects on SH-SY5Y cells, which may be considered a novel therapeutic strategy in the treatment of patients with AD.

\section{Acknowledgements}

The present study was supported by a grant from The National Natural Science Foundation of China (grant nos. 81373706, 81373619 and 81202811).

\section{References}

1. Götz J, Deters N, Doldissen A, Bokhari L, Ke Y, Wiesner A, Schonrock N and Ittner LM: A decade of tau transgenic animal models and beyond. Brain Pathol 17: 91-103, 2007.

2. Mattson MP: Pathways towards and away from Alzheimer's disease. Nature 430: 631-639, 2004.

3. Minter MR, Taylor JM and Crack PJ: The contribution of neuro-inflammation to amyloid toxicity in Alzheimer's disease. J Neurochem: Oct 28, 2015 (Epub ahead of print).

4. WimoA:Long-termeffects ofAlzheimer's diseasetreatment.Lancet Neurol: doi: http://dx.doi.org/10.1016/S1474-4422(15)00302-6.

5. Mawuenyega KG, Sigurdson W, Ovod V, Munsell L, Kasten T, Morris JC, Yarasheski KE and Bateman RJ: Decreased clearance of CNS beta-amyloid in Alzheimer's disease. Science 330: 1774, 2010.

6. Migliaccio E, Giorgio M, Mele S, Pelicci G, Reboldi P, Pandolfi PP, Lanfrancone L and Pelicci PG: The p66shc adaptor protein controls oxidative stress response and life span in mammals. Nature 402: 309-313, 1999.

7. Shan W, Gao L, Zeng W,Hu Y, Wang G, Li M, Zhou J, Ma X, Tian X and Yao J: Activation of the SIRT1/p66she antiapoptosis pathway via carnosic acid-induced inhibition of miR-34a protects rats against nonalcoholic fatty liver disease. Cell Death Dis 6: e1833, 2015.

8. Priami C, De Michele G, Cotelli F, Cellerino A, Giorgio M,Pelicci PG, Migliaccio E: Modelling the p53/p66Shc Aging Pathway in the Shortest Living Vertebrate Nothobranchius Furzeri. Aging Dis 6: 95-108, 2015 .

9. Li X, Gao D, Wang H, Li X, Yang J, Yan X,Liu Z and MaZ: Negative feedback loop between p66Shc and ZEB1 regulates fibrotic EMT response in lung cancer cells. Cell Death Dis 6: e1708, 2015.

10. Bhat SS, Anand D and Khanday FA: p66Shc as a switch in bringing about contrasting responses in cell growth: Implications on cell proliferation and apoptosis. Mol Cancer 14: 76, 2015.

11. Perrini S, Tortosa F, Natalicchio A, Pacelli C, Cignarelli A, Palmieri VO, Caccioppoli C, De Stefano F, Porro S, Leonardini A, et al: The p66Shc Protein Controls Redox Signaling and Oxidation-Dependent DNA Damage in Human Liver Cells. Am J Physiol Gastrointest Liver Physiol: Sep 3, 2015 (Epub ahead of print).

12. Sampaio SF, Branco AF, Wojtala A, Vega-Naredo I, Wieckowski MR and Oliveira PJ: p66Shc signaling is involved in stress responses elicited by anthracycline treatment of rat cardiomyoblasts. Arch Toxicol: Aug 30, 2015 (Epub ahead of print).

13. Guo X, Wu X, Ren L, Liu G and Li L: Epigenetic mechanisms of amyloid- $\beta$ production in anisomycin-treated SH-SY5Y cells. Neuroscience 194: 272-281, 2011.

14. Pena RR, Pereira-Caixeta AR, Moraes MF and Pereira GS: Anisomycin administered in the olfactory bulb and dorsal hippocampus impaired social recognition memory consolidation in different time-points. Brain Res Bull 109: 151-157, 2014.

15. Sorg BA, Todd RP, Slaker M and Churchill L: Anisomycin in the medial prefrontal cortex reduces reconsolidation of cocaine-associated memories in the rat self-administration model. Neuropharmacology 92: 25-33, 2015. 
16. Dubue JD, McKinney TL, Treit D and Dickson CT: Intrahippocampal Anisomycin Impairs Spatial Performance on the Morris Water Maze. J Neurosci 35: 11118-11124, 2015.

17. Hardy J and Selkoe DJ: The amyloid hypothesis of Alzheimer's disease: Progress and problems on the road to therapeutics Science 297: 353-356, 2002.

18. Zou G, Du X, Duan T and Liu T: Application of a NotI subtraction and methylation specific genome subtractive hybridization technique in the detection of genomic DNA methylation differences between hydatidiform moles and villi. Mol Med Rep 7: 77-82, 2013.

19. Chen Q, Qiu C, Huang Y, Jiang L, Huang Q, Guo L and Liu T: Human amniotic epithelial cell feeder layers maintain iPS cell pluripotency by inhibiting endogenous DNA methyltransferase 1. Exp Ther Med 6: 1145-1154, 2013.

20. Berry A, Greco A, Giorgio M, Pelicci PG, de Kloet R, Alleva E, Minghetti L and Cirulli F: Deletion of the lifespan determinant p66(Shc) improves performance in a spatial memory task, decreases levels of oxidative stress markers in the hippocampus and increases levels of the neurotrophin BDNF in adult mice. Exp Gerontol 43: 200-208, 2008.
21. Berry A, Capone F, Giorgio M, Pelicci PG, de Kloet ER, Alleva E, Minghetti L and Cirulli F: Deletion of the life span determinant p66Shc prevents age-dependent increases in emotionality and pain sensitivity in mice. Exp Gerontol 42: 37-45, 2007.

22. Berry A and Cirulli F: The p66(Shc) gene paves the way for healthspan: Evolutionary and mechanistic perspectives. Neurosci Biobehav Rev 37: 790-802, 2013.

23. Sone K, Mori M and Mori N: Selective upregulation of p66-Shc gene expression in the liver and brain of aged rats. Arch Gerontol Geriatr 55: 744-748, 2012. 